J. Phys. IV France 127 (2005) 163-168

(C) EDP Sciences, Les Ulis

DOI: $10.1051 /$ jp4:2005127025

\title{
The use of selected monochromatic X-rays to induce a cascade of gamma transitions from the 31-year nuclear isomer to the 4 second isomeric state of $\mathrm{Hf}-178$
}

\author{
N.C. Zoita ${ }^{1}$, F. Davanloo' ${ }^{1}$, C.B. Collins ${ }^{1}$, J.M. Pouvesle ${ }^{2}$, S. Emura ${ }^{3}$, \\ I.I. Popescu ${ }^{4}$, V.I. Kirischuk ${ }^{5}$, N.V. Strilchuk ${ }^{5}$, T. Uruga ${ }^{6}$ and Y. Yoda ${ }^{6}$ \\ ${ }^{1}$ Center for Quantum Electronics, University of Texas, Dallas, PO Box 830688, Richardson, \\ TX 75083-0688, USA \\ ${ }^{2}$ GREMI, CNRS, Université d'Orléans, Orléans, France \\ ${ }^{3}$ Institute of Scientific and Industrial Research, University of Osaka, Osaka 567-0047, Japan \\ ${ }^{4}$ IGE Foundation, Bucharest, Romania \\ ${ }^{5}$ Institute for Nuclear Research, Kiev, Ukraine \\ ${ }^{6}$ SPring-8/JASRI, Sayo-gun, Hyogo 679-5198, Japan
}

\begin{abstract}
The Hf-178m2 nuclear spin isomer stores $2.45 \mathrm{MeV}$ of energy for a half life of 31 years. Unperturbed, such nuclei radiate away the stored energy through the emission of gamma photons from electromagnetic (EM) transitions occurring within the nuclei. It has been shown that the irradiation of samples containing such nuclei with pulsed X-rays can accelerate the rate of the EM transitions by relaxing the selection rules upon changes of angular momenta. To date, most work has been done with incident $\mathrm{X}$-ray energies between 9 and $10 \mathrm{keV}$, and in such cases the acceleration of the rate of gamma emission is immediate. Reported here is a channel for deexcitation excited by more energetic X-rays that results in a cascade of gamma transitions that includes a 4 second statistical time lag. This more protracted release of the energy stored in samples of the Hf-178m 2 nuclear isomers encourages consideration of potential mechanical and thermal applications.
\end{abstract}

\section{INTRODUCTION}

The motions of charges ${ }^{1}$ within the nucleus are quantized and so are able to store electromagnetic (EM) excitation by promoting one or more of the constituent nucleons up the resulting ladders of excited states. Interactions leading to spontaneous emission of the excess energy are strong and lifetimes of excited states are short. In nuclei the spins of the component particles are usually "paired" in antiparallel combinations. A nuclear spin isomer results when such a pair is "broken" and rearranged so that the two individual spins are parallel. Spontaneous EM emission may then be inhibited by selection rules limiting changes in total angular momentum and its projections and lifetimes against spontaneous decay reach values of seconds to millions of years. In Hf-178 two isomeric states are important, "m1" which results from one broken pair and has a half life of 4 seconds, and " $\mathrm{m} 2$ " formed from two broken pairs and having a half life of 31 years. The latter stores $2.45 \mathrm{MeV}$ of excitation energy and the combination of such a high energy with such a long "shelf-life" makes the Hf-178m2 nuclear spin isomer a particularly attractive candidate for potential applications ranging from a gamma ray laser to compact sources of motive power.

The energy levels of Hf-178 are arranged into at least 19 bands that resemble the rotational bands of molecules, but only the two shown in Fig. 1 are involved in the spontaneous decay of the Hf-178m2 nuclear isomer through the EM transitions shown by the arrows. Individual levels are labeled by the total

\footnotetext{
${ }^{1}$ Neutrons act as negative "holes" in the average positive fluid of the nucleus.
} 


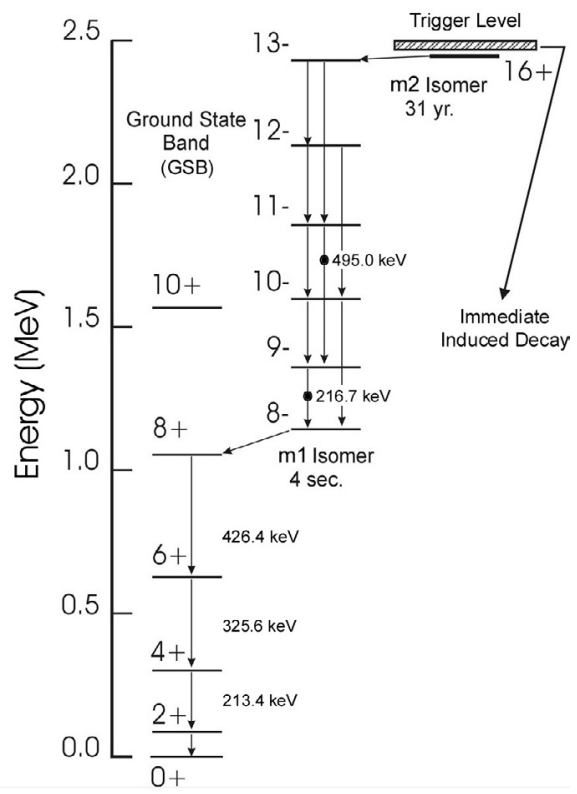

Figure 1. Energy levels of Hf-178 involved in spontaneous EM decay.

spin of the state and transitions that yield prominent lines in the gamma spectrum are labeled by the energies of the photons emitted.

In 1999 it was reported that pulsed Bremsstrahlung X-rays could accelerate the emission of gamma photons from such isomeric nuclei, so that some of the stored energy could be released upon demand [1]. The induced gamma rays were emitted from discrete transitions that followed the normal cascades of spontaneous decay. The most intense induced emission was found in the line at $495.0 \mathrm{keV}$ shown in Fig. 1. As seen there the continuation of that cascade of transitions would populate the 4 second isomeric state and so "spread" the duration of the induced energy release over a time scale more attractive to potential mechanical and thermal applications. However, there were also gamma photons from spectral lines not observed in spontaneous decay [2]. In 2001 tunable monochromatic X-rays from the synchrotron radiation (SR) source, SPring- 8 were used to determine the most favorable X-ray energies for inducing those "new" gamma lines. It was found that they were excited by energy transfer from the inner shell electrons of the Hf [3]. Incident X-rays created L3 shell vacancies and then $0.16 \%$ of the events quenching those ions transferred the energy that was released into further nuclear excitation of the " $\mathrm{m} 2$ " isomeric state to a "Trigger level" as seen in Fig. 1. The 31-year lifetime of the Hf- $178 \mathrm{~m} 2$ isomeric state is the result of a high degree of alignment of the spins of the constituent nucleons and the extra energy and angular momentum received from the quenching of the electron vacancies, relaxed the selection rules that had inhibited spontaneous electromagnetic decay. The "new" cascades of gamma photons from transitions not involved in spontaneous decay bypassed the $8^{-}$band with its statistical time lag of $4 \mathrm{~s}$ and the stored energies of the isomeric nuclei were released immediately [4]. In contrast to the first reports that the stored energy could be released upon demand, the triggered release mediated by X-rays favoring the photoionization of L3 shell electrons encouraged potential applications benefiting from the controlled release of high levels of power, as opposed to energy. Such triggered release of high power could be significant for the realization of a gamma ray laser [4]. We report here that there is a choice between the slow release of energy stored in the Hf- $178 \mathrm{~m} 2$ nuclear isomer and the immediate release already reported [4]. The slow release through the 4 second statistical lag of the $8^{-}$level is induced by monochromatic X-rays having energies near $20,825 \mathrm{eV}$, for example. 


\section{EXPERIMENTAL METHOD}

The experimental method has been detailed recently [4] and will be summarized only briefly for convenience. To optimize performance, particular care in characterization of the physical target and its alignment was essential. Targets typically contained about $1.8 \times 10^{13}$ isomeric nuclei. The isomeric fraction was about $10^{-3}$ of the total $\mathrm{Hf}$ content in the form $\mathrm{HfClxOy}$. Also included in the material from which the samples were fabricated were comparable levels of activity from an impurity, Hf-172 and its daughters. Each sample was encapsulated and mounted on a goniometer to be inclined about $7^{0}$ above the plane of propagation of the incident X-rays from the SR source. In this way the normal area presented by the target to the SR flux was smaller that the transverse cross section of the SR beam. The BL01B1 beamline at SPring- 8 was used to produce fluxes that ranged from $9.5 \times 10^{10}$ to $2.0 \times 10^{12}$ photons cm ${ }^{-2}$ $\mathrm{s}^{-1}$ with nominal spectral bandwidths of $1 \mathrm{eV}$.

Spectra of the gamma photons emitted from the samples were examined while advancing the energy of the X-rays in steps with data being collected for periods selected from a range from 100 to $500 \mathrm{~s}$ for each step. Often data were collected for an additional 30 to $150 \mathrm{~s}$ during which the SR was blocked by a thick absorber. The SR intensities and sample absorption coefficients were continuously measured in-line with ionization chambers placed before and after the isomeric target. Two opposed Ge detectors $47 \mathrm{~mm}$ thick $\times 45 \mathrm{~mm}$ dia. and $43 \mathrm{~mm}$ thick $\times 50 \mathrm{~mm}$ dia., respectively, were placed $22 \mathrm{~mm}$ from the target at right angles to the beam with the target between them. Each was shielded with layered foils of $\mathrm{W}, \mathrm{Cd}$, and $\mathrm{Cu}$, as seen by the beam. They were connected to EG\&G 673 spectroscopy amplifiers from which the output was continuously digitized and recorded to permit subsequent reexamination of the electrical pulses describing the detected photons. Data were analyzed with a mesh of $6.8 \gamma$ channels per keV. When the SR monochromator changed energies, a digital signal was sent to a third channel of the data logging system to correlate the signals from $\gamma$ photons with the energies of the irradiation. Regions of interest (ROI's) associated with each of the spectral features were identified in the 4096 channel histograms of the numbers of $\gamma$ photons detected during each period of irradiation with SR.

\section{RESULTS}

Figure 2 shows the additional numbers of gamma photons detected during an experiment conducted in 2002 when a target was irradiated with a flux of $2.83 \times 10^{11}$ photons $\mathrm{cm}^{-2} \mathrm{~s}^{-1}$ for 100 seconds in three identical consecutive runs at an X-ray energy near $20,825 \mathrm{eV}$. Also shown for comparison is a calibrated fraction of the spontaneous emission collected in the same time. In agreement with the first report [1] of an acceleration of the decay of Hf- $178 \mathrm{~m} 2$ isomeric nuclei caused by incident X-ray pulses, the enhancement of the number of photons emitted the line at $495.0 \mathrm{keV}$ in the gamma spectrum is pronounced. These data were taken during a survey mode of operation in which broad ranges of X-ray energies had to be traversed in the limited irradiation time available and the scatter seen in the data reflects that approach.

Figure 3 shows the excitation function for the induced decay of the isomeric nuclei. The sums of the numbers of extra gamma photons caused by the SR irradiation have been plotted as functions of the incident monochromatic X-rays. The transition emitting the line at $216.7 \mathrm{keV}$ is identified in Fig. 1 and the designation "3 lines" refers to the sums of counts from photons from the transitions in the ground state band (GSB) at 213.4, 325.6, and $426.4 \mathrm{keV}$. In this experimental series the X-ray energies were increased with time in the steps seen.

From Fig. 1 is can be seen that the strong enhancement of the transitions of the $8^{-}$band must be expected to deliver increased population to the band head, "m1" isomeric state. Consequently, the enhancements shown in Fig. 3 from the three lines of the GSB must have been delayed by a statistical time lag of 4 seconds. There was a nominal two second delay associated with the stepping of the X-ray energy to a higher value during which the X-ray flux was not interrupted. As a result, at the beginning of 


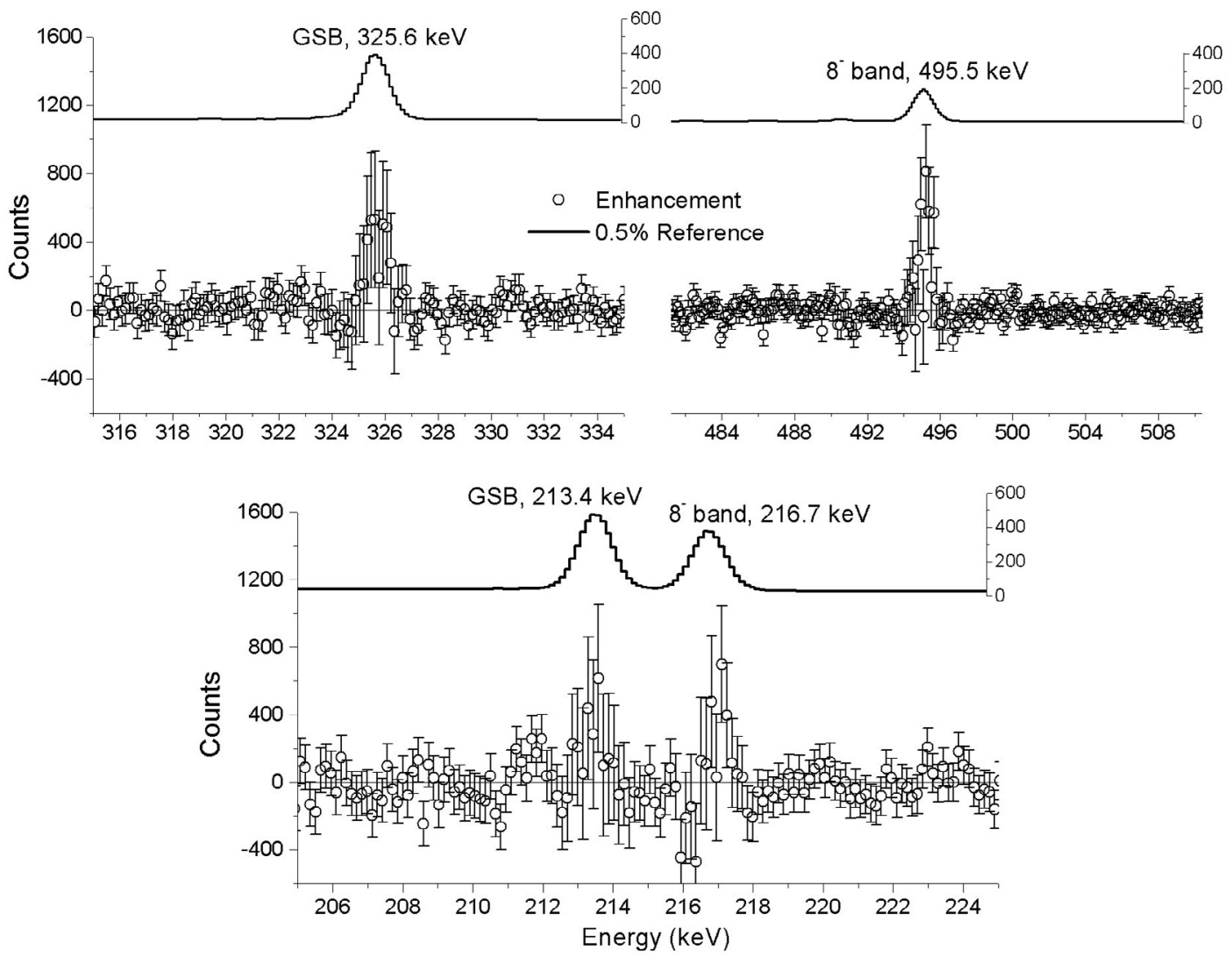

Figure 2. Enhancement of members of the $8^{-}$and GSB.

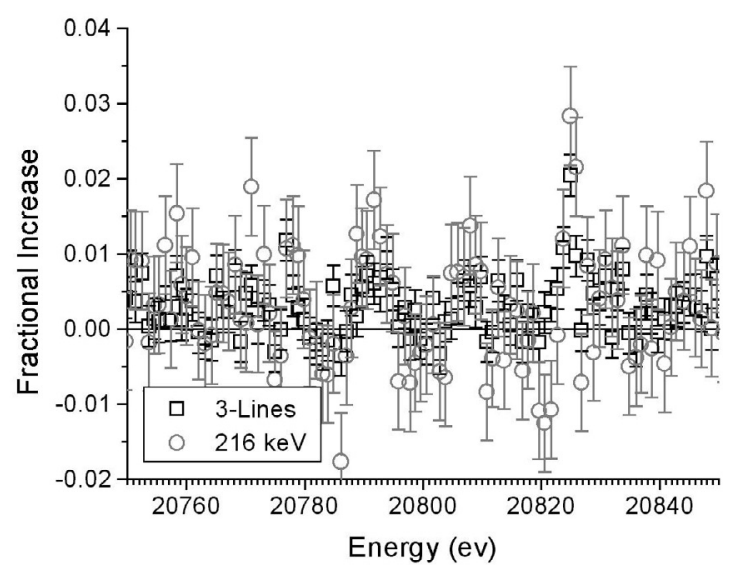

Figure 3. Excitation function for the induced decay of the Hf- $178 \mathrm{~m} 2$ isomeric state.

the period for collection of the extra numbers of counts from the detected gamma photons there should have been more in ROI's identified with members of the $8^{-}$band than from the GSB if the X-ray energies were being stepped to values for which the probability of triggering was increasing. 
Figure 4 shows the time dependence of the ratio of the numbers of induced counts detected in the $8^{-}$ band to those collected in the GSB during the times the X-ray energies were being stepped from 20,820 $\mathrm{eV}$ to $20,825 \mathrm{eV}$. In Fig. 3 it can be seen that the excitation function is increasing sharply over that range of values. The resulting delay in the detection of the greater numbers of GSB photons resulting from the increased efficiency for inducing the decay of the isomeric nuclei causes an apparent excess in the ratio of photons from members of the $8^{-}$band. That excess is reduced with time as the emission of members of the GSB "catches up." The data of Fig. 4 suggest that the time scale for equilibration of the emissions of induced photons in the $8^{-}$and GS bands is of the order of 4 seconds, as expected.

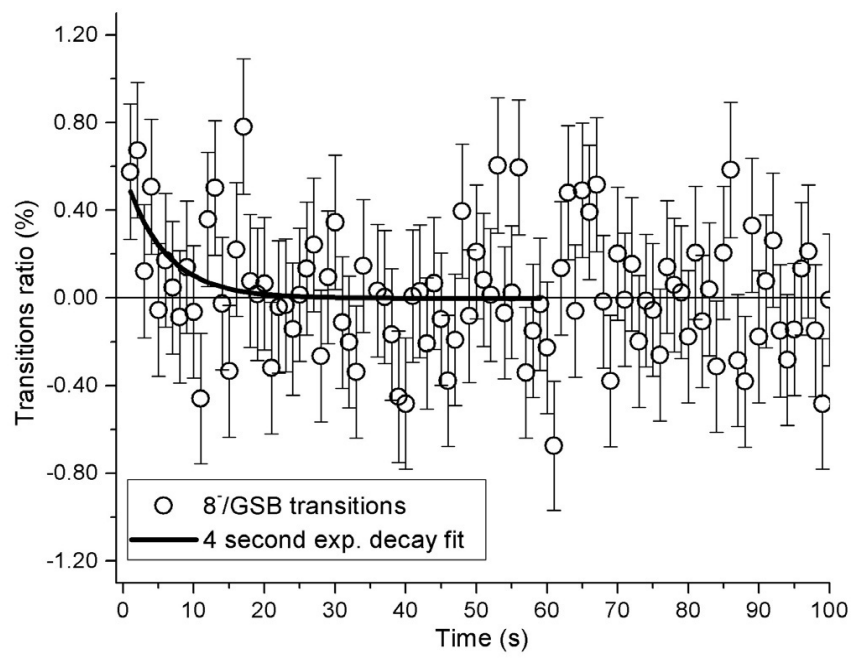

Figure 4. Exponential decay fit $\left(T_{1 / 2}=4 \mathrm{~s}\right)$ of the time dependence of the ratio of induced emission of the $8^{-}$band to GSB members during irradiation with 20,820 - 20,825 eV X-ray photons.

\section{CONCLUSIONS}

The principal conclusion of the work reported here is that there is more than one mechanism through which the irradiation with X-rays of a sample containing isomeric nuclei of Hf- $178 \mathrm{~m} 2$ can accelerate the emission of gamma photons from it. Results depend upon the distributions of energies of the incident $\mathrm{X}$-rays. Two examples have now been distinguished by the use of tuned monochromatic X-rays from SR sources. In the first case [2-4] with the use of X-rays with energies optimized for the creation of vacancies in the inner L3 shell electrons surrounding the isomeric nuclei, the onset of accelerated gamma emission is immediate. It is too fast to resolve with the timing instruments available. Triggering the release of the stored EM with X-rays in that range of $9-10 \mathrm{keV}$ may be important to applications depending upon high powers, as opposed to just the release of high energies. In the second case, the use of $\mathrm{X}$ rays with energies around $20,825 \mathrm{eV}$ causes the accelerated emission of gamma photons from many of the transitions detected in the unperturbed spontaneous decay. As in spontaneous decay, the induced emission of gamma photons belonging to members of the GSB are delayed by the 4 second statistical time lag associated with cascades that pass through the " $\mathrm{m} 1$ " isomeric level. Motive applications in which only the released energy is to be optimized would be favored in this second case in which the effects of triggering are more protracted. From the paucity of sampled X-ray energies, no conclusions can be advanced about the extension of these two distinct cases to other potential X-ray energies for accelerating the decay of the Hf-178m2 isomeric nuclei. Further measurements are planned in order to explore the pervasiveness of the two types over the range of X-ray energies which might be arranged in devices of practical scale. 


\section{Acknowledgments}

The authors gratefully acknowledge the support of AFOSR Grant No. F49620-02-1-0141 in cooperation with Japan Synchrotron Research Institute (JASRI) through proposal 2002A0321-NX-np.

\section{References}

[1] Collins C.B. et al., Phys. Rev. Lett. 82, 695 (1999).

[2] Collins C.B. et al., Phys. Rev. C 61, 054305 (2000).

[3] Collins C.B. et al., Europhys. Lett. 57, 677 (2002).

[4] Collins C.B. et al., Laser Phys. 14, 154 (2004). 